\title{
Estimating Shear-Wave Splitting Parameters from Broadband Recordings in Japan: A Comparison of Three Methods
}

\author{
by Maureen D. Long and Rob D. van der Hilst
}

\begin{abstract}
The goal of this study was to evaluate the performance of different splitting measurement techniques in the particularly complicated tectonic setting of subduction beneath Japan. We use data from the broadband Japanese $F$-net array and consider the methods of Silver and Chan (1991), Levin et al. (1999), and Chevrot (2000). We find that the results generally agree well, although discrepancies arise if the anisotropy beneath the station is more complex than the simple single-layer anisotropic model often assumed in splitting studies. A combination of multichannel and single-record methods may serve as a powerful tool for recognizing complexities and for characterizing upper-mantle anisotropy beneath a station.
\end{abstract}

\section{Introduction}

Measurements of seismic anisotropy, a phenomenon in which the velocity of a seismic wave depends on its polarization or propagation direction, can potentially illuminate important questions about deformational processes in the Earth. Upon propagation through an anisotropic medium, a shear wave will be split into a fast and a slow component and will accumulate a delay time between the orthogonally polarized components (Keith and Crampin, 1977). The fast direction $\varphi$ represents the polarization direction of the fast shear wave, and the delay time $\delta t$ represents the time difference between the fast and the slow arrivals. If the relationships between tectonic processes and strain and between strain and anisotropy are known, then shear-wave splitting measurements can provide us with information about causes, mechanisms, and consequences of deformation in the Earth (Silver, 1996; Park and Levin, 2002). Studies of uppermantle anisotropy (and its relationships with deformation and tectonics) from shear-wave splitting observations have become popular in the last two decades (e.g., Ando et al., 1983; Silver and Chan, 1988; Vinnik et al., 1989; Savage and Silver, 1993). Splitting observations are an unambiguous indicator of anisotropy (Babuška and Cara, 1991) and avoid the inherent trade-off between anisotropy and lateral wavespeed heterogeneity of direction-dependent travel-time measurements and surface-wave studies (e.g., Forsyth, 1975; Simons et al., 2002). However, the measured splitting parameters for a single shear phase represent a path-integrated picture of anisotropy; without additional information it is not possible to infer where along the path the anisotropy is located.

Although shear-wave splitting studies are a powerful and popular method for characterizing upper-mantle anisotropy and deformation, the measurement of splitting param- eters from broadband recordings of shear phases is not entirely straightforward. Typical split times due to uppermantle anisotropy average $\sim 1 \mathrm{sec}$ and range up to 2.0-2.5 sec (Savage, 1999); this is much smaller than the characteristic periods of phases used to probe upper-mantle anisotropy (core-refracted $S K S$-type, and teleseismic direct $S$ phases). Therefore, the split shear waves usually do not achieve a clear separation on the horizontal components. Additionally, most shear-wave splitting measurement methods rely on the simplifying assumption that the measured phase has passed through a single anisotropic layer with a horizontal axis of symmetry. If this condition is violated, then the measured splitting parameters are merely apparent measurements and their variation with respect to parameters such as backazimuth and angle of incidence must be examined.

Several different methods have been developed to measure shear-wave splitting parameters from broadband records. Silver and Chan $(1988,1991)$ developed a method that measures splitting parameters from a single horizontalcomponent recording that grid-searches for the best-fitting $(\varphi$, $\delta t$ ) that produces the most nearly singular covariance matrix between the splitting-corrected horizontal components. A similar method, based on maximizing the cross-correlation between corrected components, has been used by Fukao (1984), Bowman and Ando (1987), and Levin et al. (1999). Recently, Chevrot (2000) developed a method that estimates the splitting parameters $(\varphi, \delta t)$ from the relative amplitudes of the radial and transverse seismogram components as a function of incoming polarization angle. In addition to the different measurement methods used, shear-wave splitting studies often differ in key aspects of data processing, such as filtering schemes, windowing of the seismic phase of interest, and treatment of the instrument response. 
This study was motivated by observations of several researchers (e.g., Levin et al., 1999, 2004; Menke and Levin, 2003) that different measurement methods can give substantially different results; these differences have been attributed in part to complex anisotropic structure beneath the stations (in this article, we use the term "complex anisotropy" to describe any anisotropic structure that is more complicated than a single, laterally homogenous, horizontal anisotropic layer). Most comparisons among measurement methods have relied on synthetic data or have been fairly qualitative. Here, we evaluate the performance of different methods when applied to broadband seismic data from two stations of the $F$-net network in Japan, a particularly complicated tectonic region. Studies of shear-wave splitting are becoming increasingly common, but the performance of splitting measurement techniques are usually not evaluated in detail before their application to complex regions. Our aim here is to evaluate differences among splitting measurement methods in this particularly complex region before these splitting techniques are applied across the rest of the array.

Studies of shear-wave splitting due to upper-mantle anisotropy most commonly use core-refracted shear phases such as $S K S, P K S$, and $S K K S$. Core-refracted phases have two important advantages for shear-wave splitting. First, there is no contamination from near-source anisotropy; splitting must be due to anisotropic signal between the coremantle boundary (CMB) and the receiver. Second, the initial polarization of the incoming wave is constrained by the $P$ to- $S V$ conversion at the CMB. The nearly vertical incidence of $S K S$-type phases severely limits the depth resolution of splitting measurements, however, and the backazimuthal coverage at individual stations for $S K S$-type phases is often poor. These disadvantages can be ameliorated by the addition of direct $S$ phases from teleseismic events; however, contamination from source-side anisotropy must then be assessed and accounted for.

In this study we examine shear-wave splitting at two stations from F-net, a network of broadband stations in Japan. This is part of a larger effort to characterize anisotropy and mantle deformation beneath Japan using shear-wave splitting measurements (Long and van der Hilst, 2005). The primary objective of this article is to evaluate and compare different shear-wave splitting measurement methods applied to real seismic data in a complicated region. Both stations have been in operation since 1995 and provide clean, highquality data; additionally, the data coverage with respect to backazimuth and/or incoming polarization azimuth and incidence angle is excellent. We wish to answer several questions. For ideal stations, with good data coverage and signalto-noise ratio, do different splitting methods yield the same best-fitting splitting parameters? Do different methods give us consistent insight into the nature of anisotropy beneath the station? Can we achieve reliable shear-wave splitting measurements in a complex region and be sure that our characterization of anisotropy is not dependent on the measure- ment method used? What are the advantages and disadvantages of each method? Can different measurement methods yield ambiguous or even contradicting results? If so, what are the implications for the inferred anisotropic models? Do the methods respond differently to changes in preprocessing procedure, such as the use of different filtering schemes?

In designing this study, we have chosen a subset of available splitting methods to evaluate. Many methods have been developed to constrain anisotropic models beneath seismic stations, such as those of Wolfe and Silver (1998), Rumpker and Silver (1998), Restivo and Helffrich (1999), and Menke and Levin (2003). We have selected three measurement methods that make relatively few assumptions about the anisotropic structure beneath the station, and we test if a consistent picture of anisotropy emerges with each of the measurement methods and if the use of different methods can lead to different and useful insights.

\section{Splitting Measurement Methods}

The Silver and Chan (1991) Method

(Hereinafter SC1991)

This method operates upon the principle that the bestfitting splitting parameters $\varphi$ and $\delta t$ correspond to a certain inverse splitting operator that best linearizes the $S$-wave particle motion when the effect of the anisotropy is removed. In order to find the best splitting parameters, a grid search over possible $(\varphi, \delta t)$ values was performed. Vidale (1986) demonstrated that the inverse splitting operator can be found from the time-domain covariance matrix of the horizontal particle motion. If the (orthogonal) horizontal components make angles of $\varphi$ and $\varphi+\pi$ with the wave's polarization vector $\mathbf{p}$, and the time lag is given by $\delta t$, the covariance matrix $C_{i j}$ between the (orthogonal) horizontal components $u$ is given by

$$
\mathbf{C}=C_{i j}(\varphi, \delta t)=\int_{-\infty}^{\infty} u_{i}(t) u_{j}(t-\delta t) d t ; i, j,=1,2 .
$$

A quantitative measure of the linearity of the particle motion is given by the eigenvalues of $\mathbf{C}$. For the isotropic case, the $S$-wave particle motion is linear and $\mathbf{C}$ will have one nonzero eigenvalue; in the presence of anisotropy, $\mathbf{C}$ will have two. Therefore, the inverse splitting operator that best corrects for the presence of anisotropy will result in a corrected horizontal covariance matrix that is most nearly singular. The most nearly singular corrected covariance matrix is found by maximizing $\lambda_{1}$ (the larger eigenvalue), or from an equivalent eigenvalue-based measurement of linearity. Errors are estimated using a Fischer test formulation. We use a value of one degree of freedom per second; this is based on the rule of thumb given by Silver and Chan (1991) that assumes a stationary noise process and estimates the degrees of freedom directly from broadband data. 
The Cross-Correlation Method (e.g., Levin et al., 1999; Hereinafter LMP1999)

The cross-correlation method used by Fukao (1984), Bowman and Ando (1987), Levin et al. (1999), and others is very similar in concept to SC1991. This method also uses a grid search to find the pair of splitting parameters $(\varphi, \delta t)$ that corresponds to the most nearly singular covariance matrix. However, the measure of linearity used in the correlation method is not the maximization of $\lambda_{1}$ but the minimization of the determinant of the covariance matrix. This is mathematically equivalent to maximizing the cross-correlation between the horizontal components (Silver and Chan, 1991). This maximization of the cross-correlation can be visualized as searching for the inverse splitting operator that maximizes the similarity in the pulse shapes of the corrected seismogram components (Levin et al., 1999). In other words, this method tries to accommodate the prediction that the $S$ wave, after traveling through an anisotropic medium, should be composed of two orthogonally polarized pulses with identical shape, but time-delayed with respect to one other. Measurements of splitting parameters using the LMP1999 technique presented in this article were made using an implementation of the cross-correlation algorithm as presented by Levin et al. (1999). Uncertainties in the splitting parameters are computed using the metric described by these authors and are based on the deviations from perfect crosscorrelation (presumed to be caused by stochastic noise in the seismograms) for the best-fitting splitting parameters.

\section{The Chevrot (2000) Method (Hereinafter C2000)}

The above methods can be referred to as single-record methods. The multichannel method developed by Chevrot (2000) takes a very different approach to estimating splitting parameters. It is based upon the predicted variation of the amplitudes of the transverse components with incoming polarization angle (equivalent to the backazimuth for SKS-type phases). For the case where $\delta t$ is small compared to the dominant period of the waveform, the radial and transverse components for a vertically incident shear pulse with waveform $w(t)$ and polarization $\mathbf{p}$ are given by (Silver and Chan, 1988; Chevrot, 2000).

$$
\begin{gathered}
R(t) \approx w(t) \\
T(t) \approx-1 / 2(\delta t \sin 2 \beta) w^{\prime}(t),
\end{gathered}
$$

where $w^{\prime}(t)$ is the time derivative of the radial waveform $w(t)$, and $\beta$ is the angle between the fast axis $\varphi$ and the incoming polarization direction $\mathbf{p}$. In the $\mathrm{C} 2000$ method, the amplitude of the transverse component relative to the derivative of the radial component is referred to as the splitting intensity; the azimuthal dependence of the splitting intensity is referred to as the splitting function; and the splitting vector is the ensemble of estimates of the splitting function ob- tained at different incoming polarization angles represented in the data set for a certain station. When a plot of the splitting vector is produced, the best splitting parameters are estimated by fitting a $\sin (2 \theta)$ curve to the splitting vector. The amplitude of the sinusoid gives the best-fitting delay time, and the fast axis can be inferred from the phase of the sinusoid at the origin. An illustration of the variations in transverse component amplitude with incoming polarization is given in Figure 1, in which radial and transverse components for shear arrivals at $F$-net station TKA with similar incoming polarization angles have been stacked in $20^{\circ}$ bins.

\section{Data}

In this study we utilize data from two broadband stations in the $F$-net network. Station SGN is located near Tokyo, in central Honshu, and station TKA is located in southern Kyushu (Figure 2). Both stations have been in operation since 1995; the measurements presented in this article come from data collected between 1995 and 2002. We selected clean recordings of teleseismic $S$ phases (in the $40^{\circ}-80^{\circ}$ epicentral distance range), $S K S\left(85^{\circ}-120^{\circ}\right)$, and $S K K S$ (beyond $105^{\circ}$ ), as well as slab events located directly beneath the stations. An event map is shown in Figure 3. We restricted our use of direct $S$ phases to deep ( $>200 \mathrm{~km}$ ) events to reduce contamination from source-side anisotropy for teleseismic $S$ and to ensure that we sampled as much of the upper mantle as possible for local $S$. We have extensively tested our $F$-net splitting measurements to show that source-side contamination from direct teleseismic $S$ phases is not a major factor in this data set. In these tests, we looked for systematic variation in measured splitting parameters due to variations in source depth or region. With the exception of a few intermediate-depth events from the Tonga subduction zone, which were subsequently removed from the data set, we found no evidence for systematic source-side contamination. These tests are described in detail by Long and van der Hilst (2005). With the use of direct $S$ phases in addition to corerefracted phases we ensured that our data set covers a wide range of backazimuths, initial polarization azimuths, and incidence angles. Good data coverage in terms of initial polarization azimuths is necessary for the implementation of the multichannel method; additionally, good coverage in terms of all three parameters is necessary to gain sensitivity to structures more complex than a single horizontal anisotropic layer.

All of the broadband data were bandpass filtered using a 4-pole Butterworth filter with corner frequencies at 0.02 $\mathrm{Hz}$ and $0.125 \mathrm{~Hz}$. In order to implement the multichannel method, the two horizontal components were rotated by the measured incoming polarization angle to separate the radial and transverse components. For all phases the initial polarization angle (direction of maximum polarization) was measured directly from the seismogram. This was done by computing the direction of maximum polarization using a covariance measure; see Vidale (1986). Although the shear- 

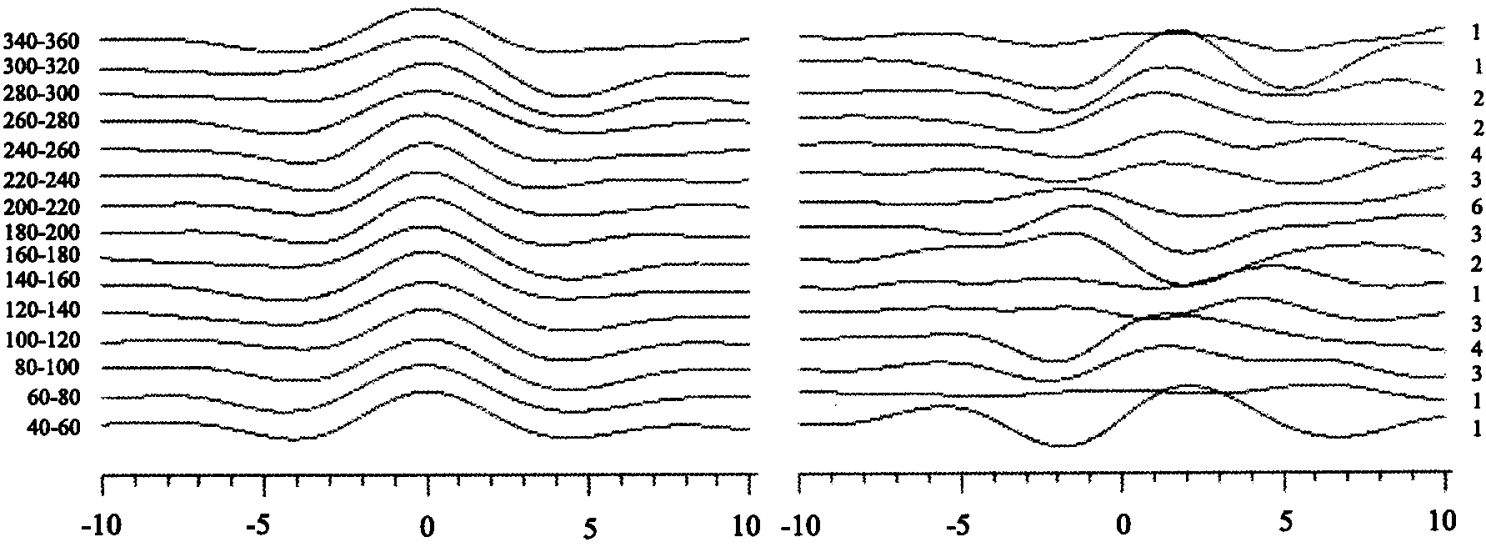

Figure 1. Illustration of the multichannel method using data from $F$-net station TKA. In the left panel, radial-component traces for a variety of incoming polarization angles are shown. Traces are stacked in $20^{\circ}$ polarization angle bins, and radial-trace amplitudes have been normalized to 1 . In the right panel, the corresponding stacked transverse traces are shown. To the left of the traces is the incoming polarization angle range; to the right of the traces is the number of records in the stack. The dependence of the transverse component on incoming polarization angle is clearly shown.

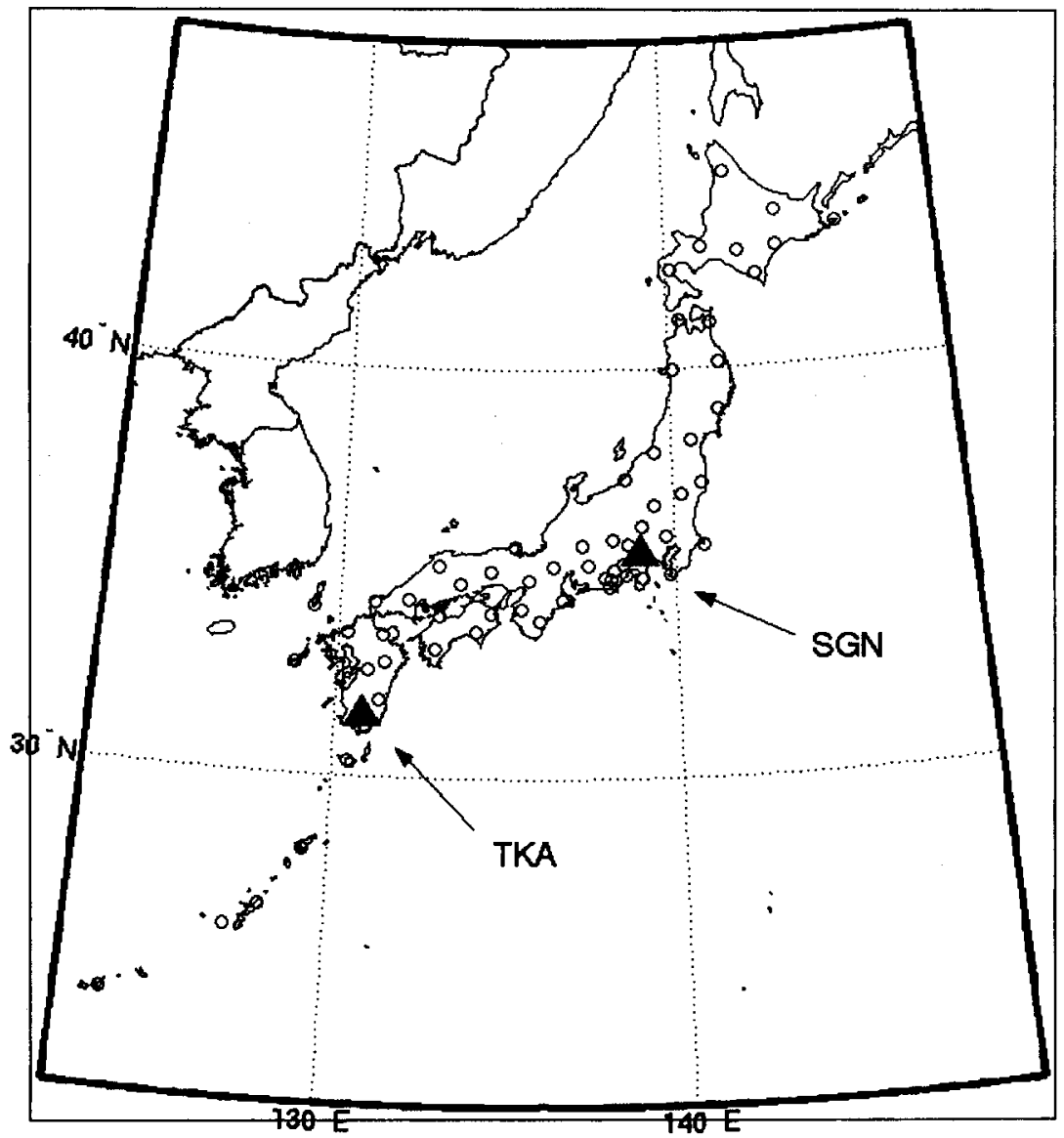

Figure 2. A station map for $F$-net. Stations in the network are marked with small circles; the two stations examined in this study, SGN and TKA, are marked with large triangles.

wave particle motions are elliptical due to the effect of splitting, the direction of maximum polarization can be retrieved because the splitting times are much smaller than the dominant period of the incoming phase. For $S K S$ and $S K K S$ phases, which generally had smaller signal-to-noise ratios than the direct $S$ phases, the measured incoming polarization angle was compared to the backazimuth. The vast majority agreed to within $10^{\circ}$, and those records that did not (due to low signal-to-noise ratio or phase misidentification) were discarded. The rotated horizontal traces were then standard- 


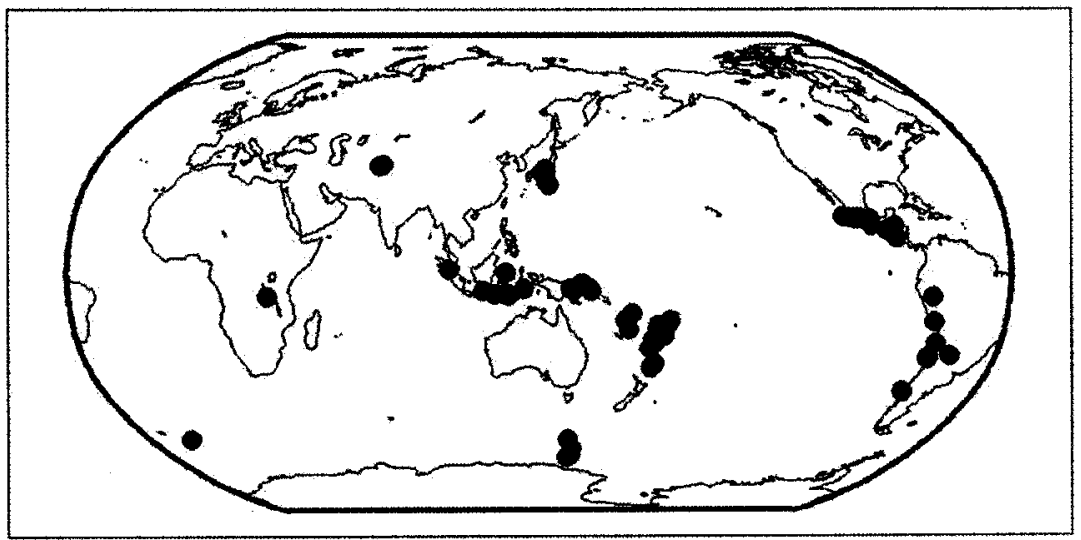

Figure 3. Local and teleseismic events used in this study.

ized by a deconvolution of the radial waveform from the radial and transverse components. For all records the shape of the transverse waveform was compared to the time derivative of the radial waveform, and traces for which the transverse waveform did not match the predicted shape were discarded. In this way, all records were visually checked for satisfactory signal-to-noise ratio and waveform clarity. Seismograms for an SKS arrival recorded at station TKA in various stages of processing are shown in Figure 4.

\section{Splitting Measurements for $F$-net Stations}

\section{Station TKA}

At station TKA we identified 36 very high quality recordings of shear phases from which we measured shearwave splitting (28 teleseismic $S, 5$ local $S, 2 S K S, 1 S K K S$ ). Measured splitting parameters from each of the three methods are shown in Figure 5; for each method, the measurements are plotted as a function of incoming polarization azimuth. The $\mathrm{C} 2000$ splitting vector exhibits a striking $\sin (2 \theta)$ dependence, although there is some scatter in the best fit to the data that is larger in amplitude than the size of the $2 \sigma$ formal error bars. However, a fit of a $\sin (2 \theta)$ curve to the splitting vector allows us to retrieve best-fit splitting parameters of $\varphi=49.9^{\circ} \pm 1.4^{\circ}, \delta t=0.60 \mathrm{sec} \pm 0.03 \mathrm{sec}$. The good fit of a $\sin (2 \theta)$ curve to the splitting vector TKA demonstrates that the C2000 method works well with the two modifications we have introduced: (1) the use of direct $S$ phases in addition to $S K S$-type phases and (2) the use of splitting intensity measurements from individual records in the construction of the splitting vector rather than stacking several measurements in each backazimuthal window. Indeed, several studies (e.g., Schulte-Pelkum and Blackman, 2003) have demonstrated that stacking or averaging splitting measurements in backazimuthal windows results in a loss of sensitivity to complex anisotropic structure.

The results from the LMP1999 and SC1991 measurements are also plotted as a function of incoming polarization azimuth in Figure 5. It should be noted that there are fewer usable measurements for these two methods than for the multichannel method. This is due to the different treatment of null splitting measurements by the two classes of method. Measurements of little or no splitting are easily incorporated using the C2000 method, because these are simply represented by splitting intensities close to zero. Null splitting is represented on the plots of single-record measurements with crosses; however, splitting parameters cannot be extracted from records with null or near-null splitting using the singlerecord measurement methods. The average split times obtained with single-record methods tend to be larger than the $\delta t$ obtained with the multichannel method; moreover, both single-record methods have a tendency to obtain unreasonably large split times for some records with small amplitudes on the transverse components [this phenomenon has been observed by several other authors, including Silver and Chan (1991) and Levin et al. (1999)]. In general, however, the three methods yield comparable average splitting measurements for station TKA, and the splitting patterns obtained with all three methods are generally consistent with a singlelayer model of anisotropy beneath station TKA.

\section{Station SGN}

Splitting results for station SGN are shown in Figure 6. At this station we obtained measurements for 21 teleseismic $S$ recordings, 3 local $S, 9 S K S$, and 5 SKKS. The splitting vector obtained with the multichannel method deviates more from a simple $\sin (2 \theta)$ variation than the vector at station TKA. We can still fit a $\sin (2 \theta)$ curve to the splitting vector and obtain splitting parameters $\left(\varphi=-48.0^{\circ} \pm 1.1^{\circ}, \delta t=\right.$ $0.57 \mathrm{sec} \pm 0.03 \mathrm{sec}$ ), but the scatter in the data suggests a deviation from simple anisotropy beneath this station. The LMP1999 results at this station (Figure 6) also exhibit intriguing complexity; the plot of measured fast directions as a function of incoming polarization angle reveals a clear $90^{\circ}$ periodicity, which is characteristic of two anisotropic layers (Özalaybey and Savage, 1994; Silver and Savage, 1994). It is difficult to discern a clear periodicity in the LMP1999 $\delta t$ measurements, however, and these measurements also deviate significantly from the predictions of a simple anisotropic model. SC1991, curiously, yields fewer stable mea- 


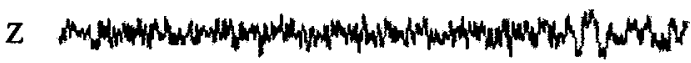

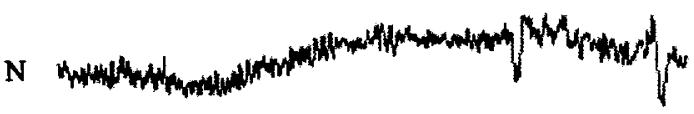

E

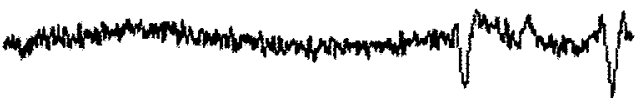
0

0

T

150

\section{0}

100

200
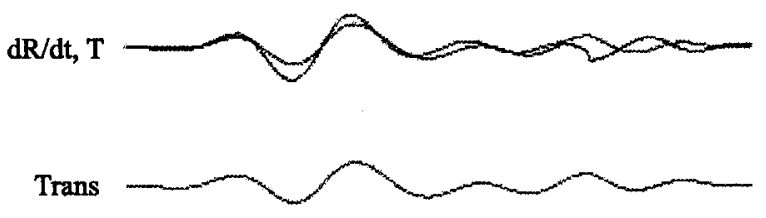

$\operatorname{Rad}$

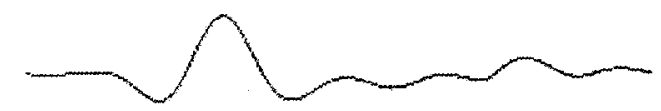

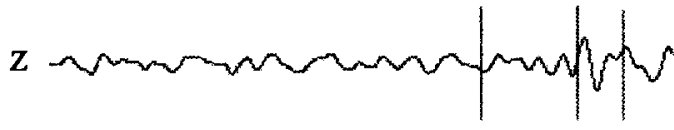

Namangangly

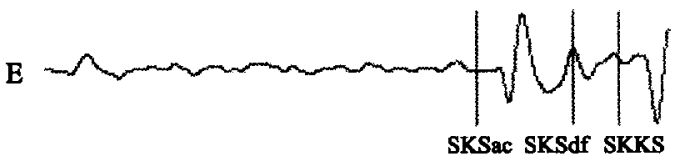

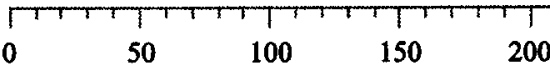

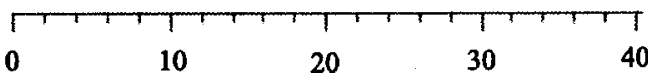

Figure 4. Record of an $S K S$ phase at station TKA in various stages of processing. At top left, a raw, unfiltered three-component seismogram is shown. At top right, the traces have been bandpass-filtered with corner frequencies at $0.02 \mathrm{~Hz}$ and $0.125 \mathrm{~Hz}$, and the expected travel times from the iasp91 earth model for $S K S$ and $S K K S$ are shown. At bottom left, the filtered horizontal traces have been rotated by the backazimuth to show the radial component (bottom trace) and transverse component (middle trace). The radial component has been deconvolved from both horizontal traces to standardize them. In the top trace, the transverse component is overlain with the time derivative of the radial component. As expected, the transverse component has the shape of the radial component derivative.

surements at SGN than the LMP1999 method, and it does not yield a $90^{\circ}$ periodicity. The average $\delta t$ obtained with SC1991 is significantly higher than the averages obtained with the other two methods, and the formal errors for individual SC1991 measurements are larger than the formal errors for LMP1999 measurements.

Can we attribute the differences in splitting pattern between stations TKA and SGN to differences in data quality or differences in source-side contamination from direct $S$ phases? We do not observe any difference in signal-to-noise ratios between the two stations; TKA and SGN are both very high quality stations, and only records with very clear, lownoise shear arrivals are retained in this study. As discussed in detail by Long and van der Hilst (2005), there is good evidence that source-side contamination from direct $S$ phases is not a large factor in this splitting data set. Additionally, we note that the source distribution for the two stations is nearly identical; therefore, the character of source-side contamination, if present, should not be different for the two stations. A difference in the anisotropic geometry beneath the two stations is therefore the most likely explanation for the observed difference in splitting pattern. Because a $90^{\circ}$ periodicity is observed at station SGN, two distinct anisotropic layers are likely present beneath this station.

\section{Comparison of Results from Three Splitting Measurements}

We now compare more directly the splitting results from the three different splitting methods at stations TKA, where splitting is generally consistent with a simple anisotropic model, and SGN, where the splitting patterns deviate significantly from predictions of simple anisotropy, and where a two-layer model may be needed to explain the data. The three methods agree very well at station TKA. It is difficult to compare directly individual measurements from the multichannel approach with those of the single-record methods, but a direct comparison of the SC1991 and LMP1999 measurements for station TKA is shown in Figure 7. We find that for every recording for which both methods yield 

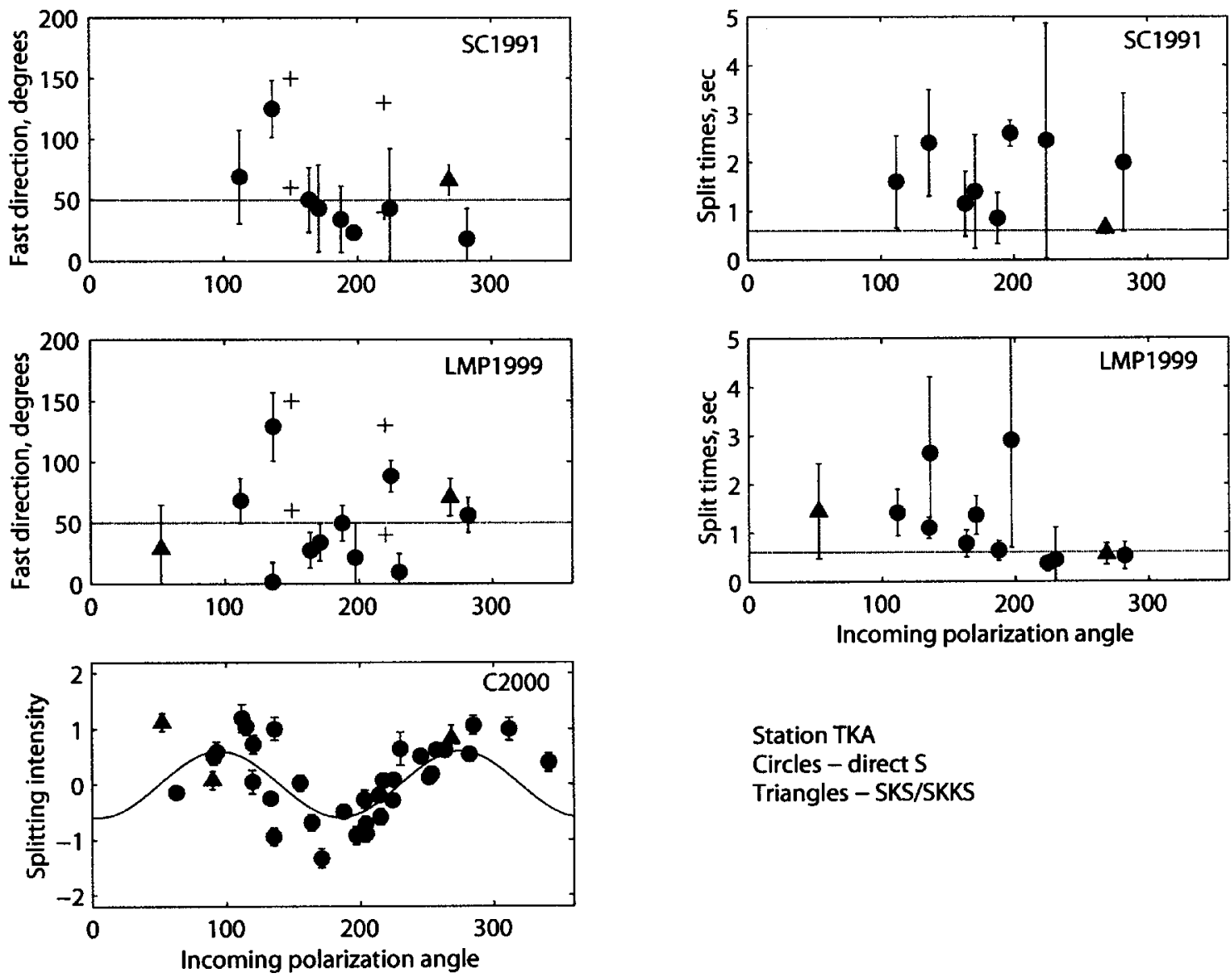

Figure 5. Results of splitting analysis for $F$-net station TKA for three measurement methods. Circles represent direct $S$ phases, and triangles represent core-refracted phases. Null measurements are shown with crosses for the SC1991 and LMP1999 methods. All measurements are plotted as a function of incoming polarization angle, which is measured directly from the seismogram for $S$ phases, and is equal to the backazimuth for $S K S / S K K S$. Polarization angles were measured between $0^{\circ}$ and $180^{\circ}$, but measured angles for events with backazimuths between $270^{\circ}$ and $90^{\circ}$ were adjusted by adding $180^{\circ}$ to the polarization angle. Therefore, arrivals with similar measured polarizations coming from events with widely different backazimuths will plot $180^{\circ}$ apart on these diagrams. All error bars are $2 \sigma$.

a usable measurement, the measurements agree within their formal $2 \sigma$ errors. (For the purpose of this comparison, we adopt the very inclusive definition that a usable measurement has a $2 \sigma$ error in the fast direction of less than $\pm 45^{\circ}$ and a $2 \sigma$ error in $\delta t$ that is less than the magnitude of $\delta t$ itself; that is, the range of $\delta t$ values allowed by the data does not include zero.) We can find some events for which the SC1991 measurement yields a more tightly constrained estimate with smaller formal errors than the LMP1999 method, but examples of the opposite can also be found.

At station SGN we also find that the average splitting parameters obtained with each method agree fairly well, with the exception of the large average $\delta t$ obtained with the SC1991 method. However, if we compare the two singlerecord methods directly (Figure 8), we see that there are several events for which the two methods yield measurements that disagree. Additionally, SC1991 yields far fewer usable measurements than the LMP1999 method. We propose that complexity in anisotropic structure (for instance, the presence of two distinct anisotropic layers) produces both the discrepancies between the two methods and the smaller number of usable SC1991 measurements. The presence of multiple anisotropic layers, and therefore multiple splitting that is measured as an apparent single-splitting measurement (Silver and Savage, 1994), probably subtly distorts waveforms such that the SC1991 method is more likely to be unstable, and such that the two methods are more likely to disagree.

We also test how the three methods respond to different filtering schemes. Several studies have found that splitting measurements can be frequency dependent (e.g., Clitheroe and van der Hilst, 1998; Matcham et al., 2000) All data presented so far in this paper were bandpass-filtered between periods of 8 and $50 \mathrm{sec}$. To assess the effects of different 

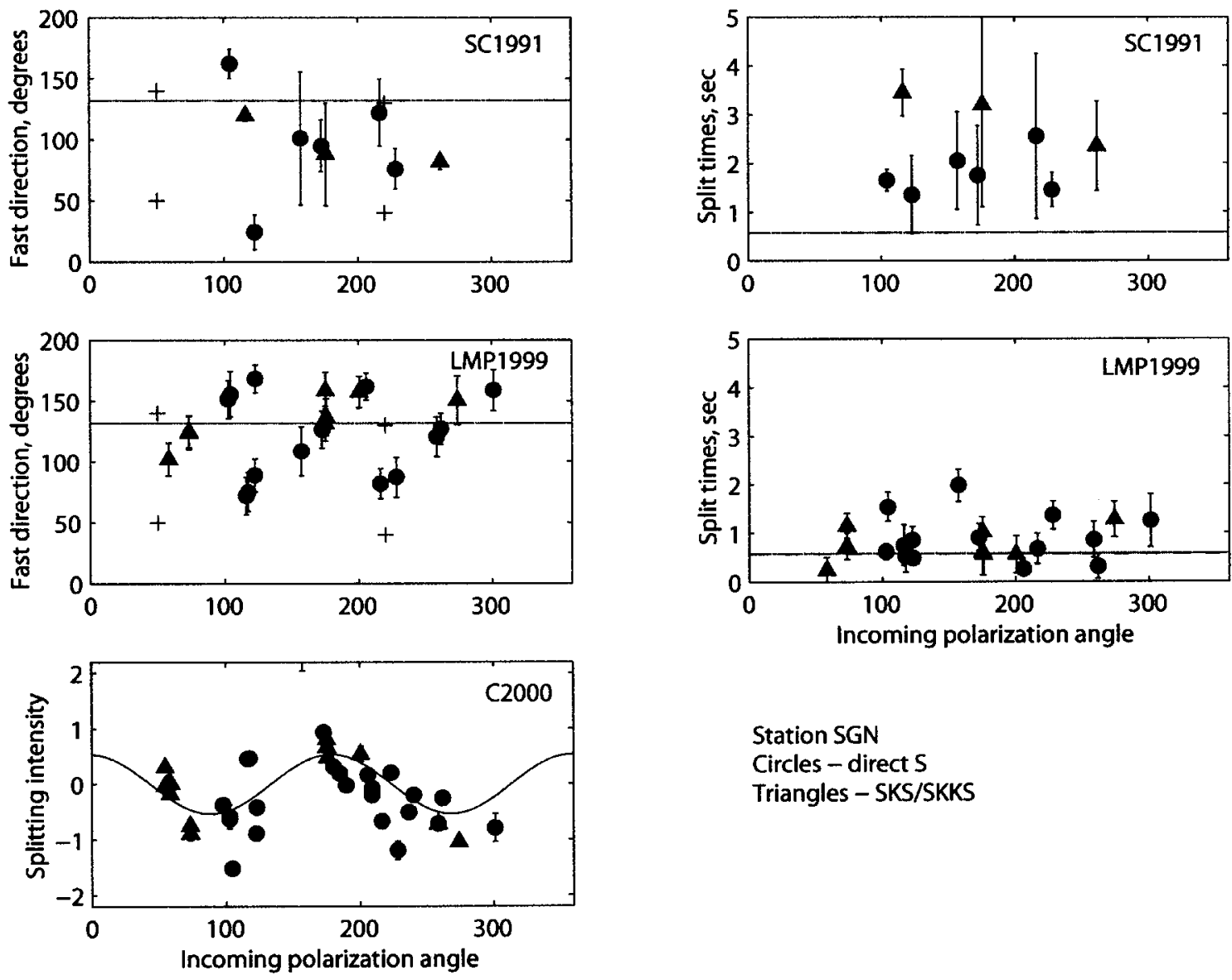

Station SGN

Circles - direct S

Triangles - SKS/SKKS

Figure 6. Similar to Figure 5, but showing results from station SGN.
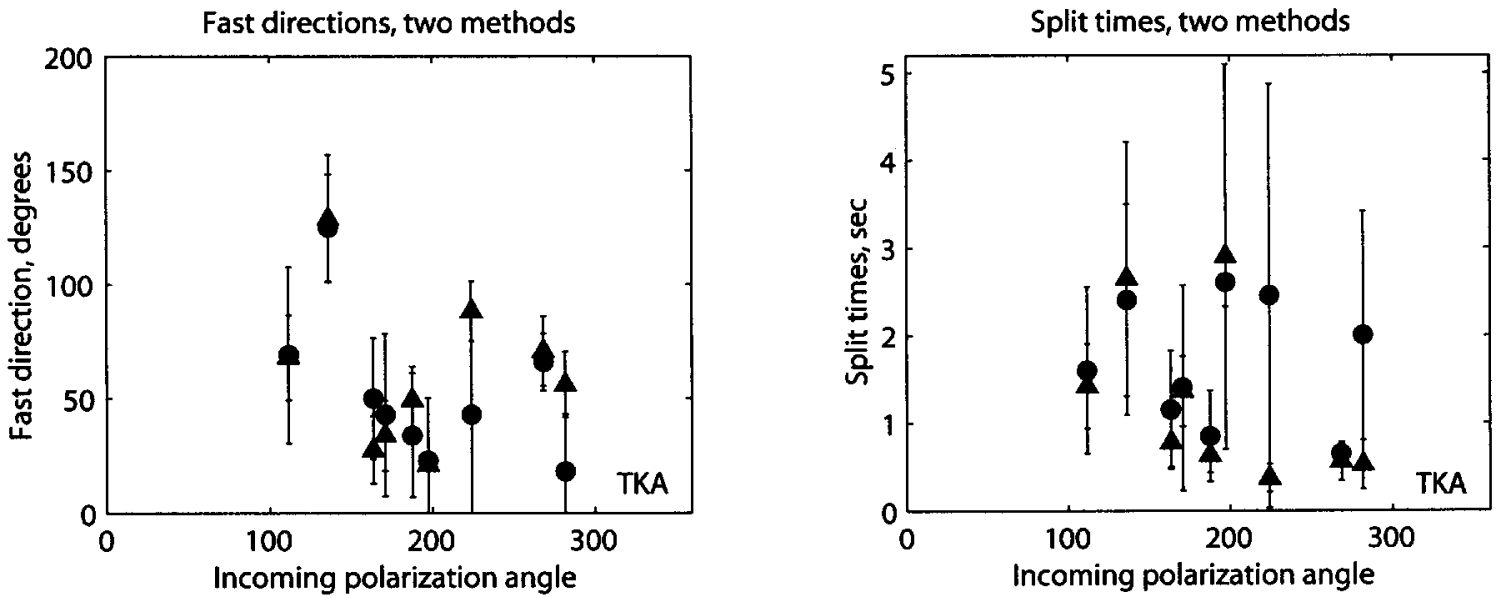

Figure 7. A direct comparison of the splitting measurements at station TKA using the so-called single-record methods. SC1991 measurements are plotted as circles, while LMP1999 measurements are plotted as triangles. Only those events which yielded a well-constrained measurement using both methods are shown on this plot. All error bars are $2 \sigma$. 

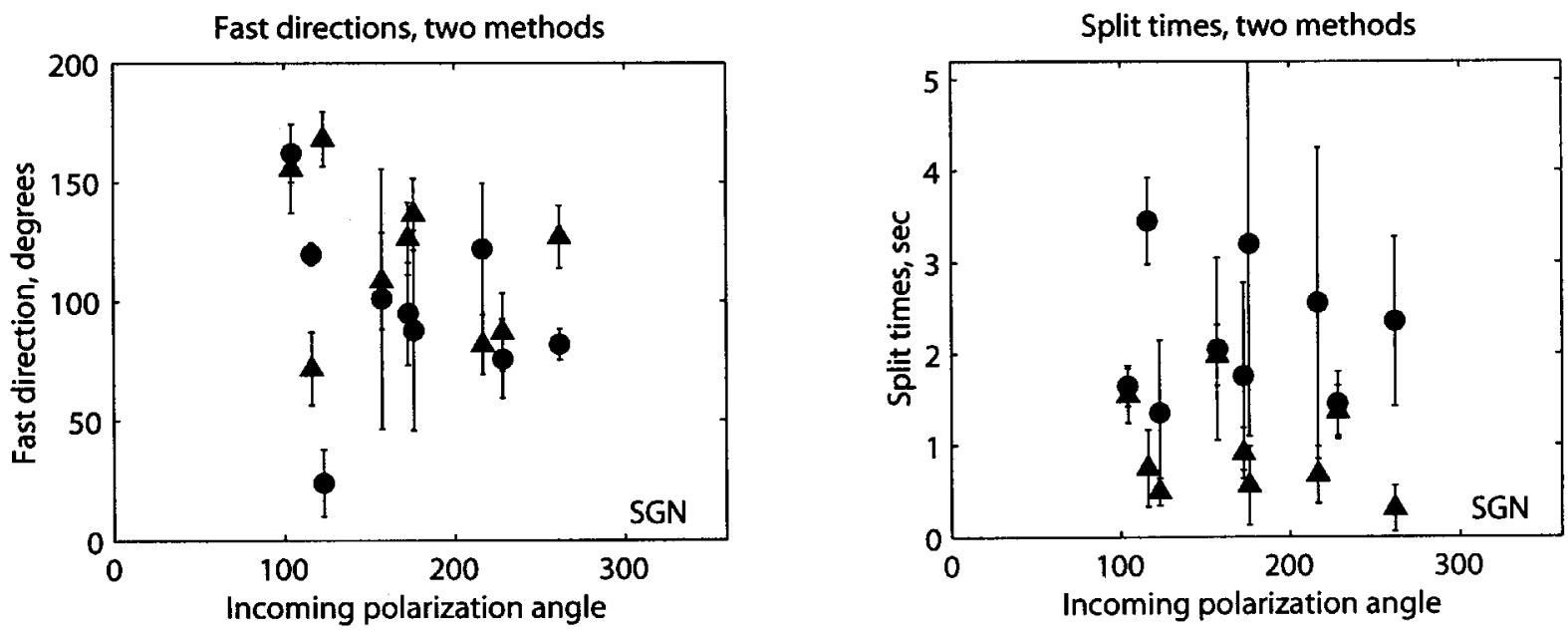

Figure 8. Similar to Figure 7, but displaying splitting measurements for station SGN.

filtering schemes on the splitting measurements, we experimented with a variety of high-, low-, and bandpass filters. Generally, we found that the measured fast direction did not depend on the filtering scheme used, and that the first-order effect on measured $\delta t$ values was to decrease the split time when primarily high-frequency signals were used. To illustrate this, we show filtering tests on TKA data for each method using high- and low-pass Butterworth filters with a corner frequency of $0.2 \mathrm{~Hz}$ (5-sec period). The results of these filter tests are shown in Figure 9. In general, fewer usable measurements were made using the high- and lowpass filtering schemes than with the bandpass filter. For the multichannel method, the best splitting parameters obtained with the low-pass filtering scheme $\left(\varphi=53.5^{\circ} \pm 2.4^{\circ}, \delta t=\right.$ $0.54 \mathrm{sec} \pm 0.06 \mathrm{sec}$ ) were consistent with the results with the original filter $\left(\varphi=49.9^{\circ} \pm 1.4^{\circ}, \delta t=0.60 \mathrm{sec} \pm 0.03\right.$ sec), while the effect of the high-pass filter was to decrease the amplitude of the sinusoid and increase the errors on the individual measurements $\left(\varphi=59.7^{\circ} \pm 7.8^{\circ}, \delta t=0.24 \mathrm{sec}\right.$ $\pm 0.07 \mathrm{sec}$ ). For the single-record methods, the most noticeable effect of the high- and low-pass filtering scheme was to dramatically decrease the number of usable measurements, making rigorous comparisons difficult. It is not obvious for the data used here that discrepancies between high-, low-, and bandpass-filtered single-record measurements are systematic. It seems that of the three methods considered here, the multichannel method is the least dependent on the filter scheme used.

\section{Discussion}

With our analysis of the performance of three splitting measurement methods at $F$-net stations SGN and TKA, we have shown that each of the methods have distinct advantages and disadvantages. The multichannel method of Chevrot (2000) provides a natural way of investigating the backazimuthal variations of splitting parameters that is associated with complex anisotropic structure, as well as a natural way of incorporating null splitting measurements into an averaging scheme. Additionally, the multichannel method is more robust against changes in frequency content than the single-record methods. However, the multichannel method has the disadvantage of requiring adequate data coverage in backazimuth (or incoming polarization azimuth for direct $S$ ), which is often impossible to achieve with $S K S$-type phases alone and can be difficult to achieve even with the use of direct $S$ phases. Also, the multichannel method requires an accurate measurement of initial polarization, which is straightforward for $S K S$-type phases but less accurate for direct $S$. The SC1991 method has the advantage of being applicable to a single record, and it does not need an accurate initial polarization measurement. Additionally, we have shown that SC1991 sometimes outperforms the LMP1999 method on individual records. However, both single-record methods are sensitive to the filtering scheme used, and both have drawbacks regarding the definition of null splitting measurements and their incorporation into averaging schemes. The SC1991 method appears to yield fewer usable measurements and larger error bars for stations that overlie complex anisotropic structures (for example, multiple layers). The LMP1999 method yielded more usable measurements than the SC1991 method at station SGN and can sometimes outperform it on individual records even for simple stations (e.g., TKA). Aside from this, it shares many of the same advantages and disadvantages of the SC1991 method.

Because each of the three methods has distinct advantages and disadvantages, and because the most time-consuming and labor-intensive part of making shear-wave splitting measurements is not in the measurements per se but rather in the data preprocessing and selection, it may be advisable to use a combination of all three measurement methods. This is especially true if the data suggest that the station is located above complex anisotropic structure, where a combination 
(a) Filtered between 8 and 50 seconds
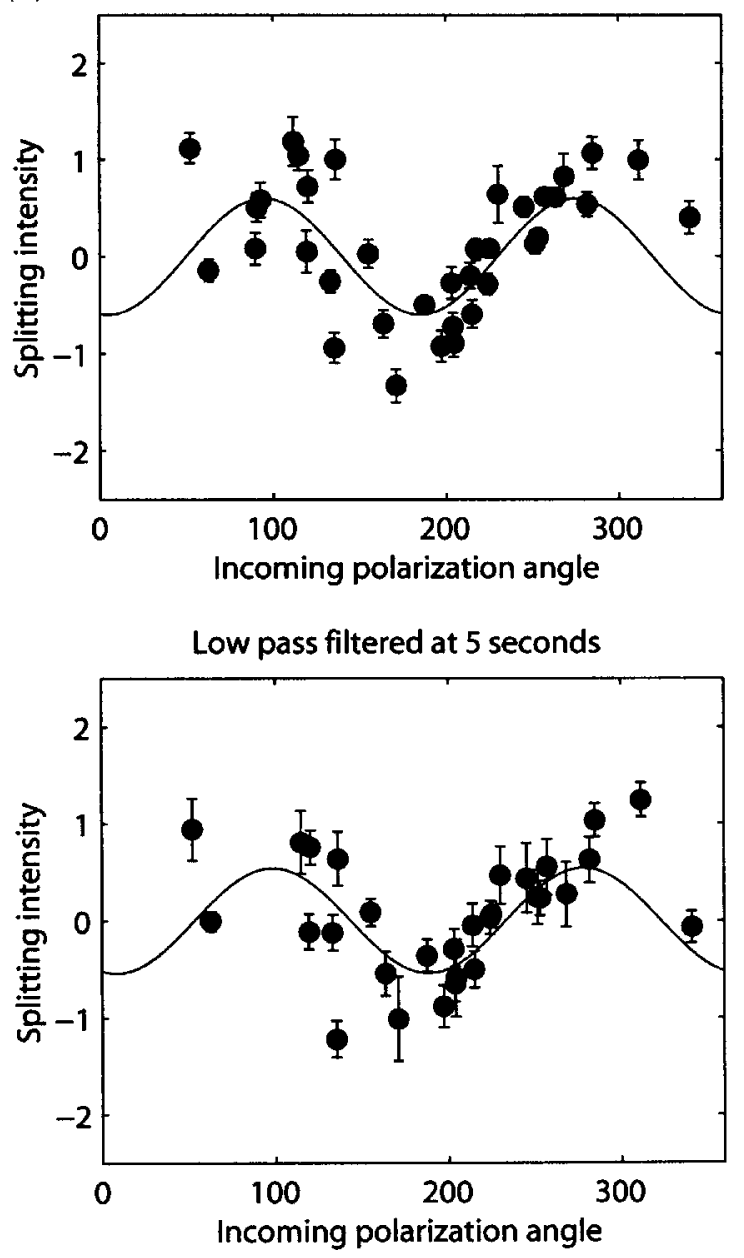

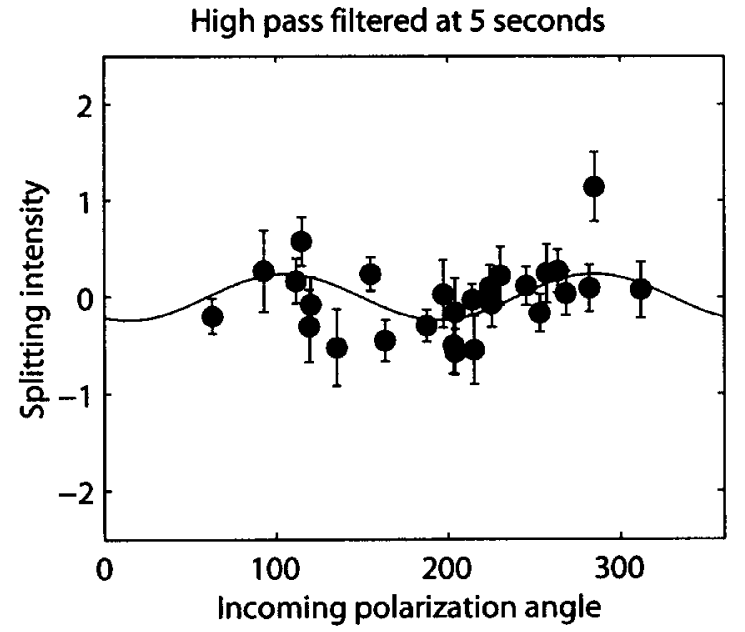

Figure 9. (a) Results of filter tests for station TKA for the C2000 method. Each plot shows the results yielded by three different filtering schemes-bandpass-filtered between 0.02 and $0.125 \mathrm{~Hz}$, high-pass-filtered at $0.20 \mathrm{~Hz}$, and low-pass-filtered at $0.20 \mathrm{~Hz}$.

(continued)

of the multichannel and single-record methods can be especially powerful. The LMP1999 method seems to outperform the SC1991 method at stations overlying complex anisotropic structures, but SC1991 can occasionally yield better-constrained estimates than the LMP1999 method for individual records. Therefore, the application of both the SC1991 and LMP1999 methods increases the number of usable measurements at a given station.

In situations where the anisotropic structure beneath the station is complex, the average splitting parameters obtained either by taking a weighted average of individual singlerecord measurements or by fitting a $\sin (2 \theta)$ curve to the multichannel splitting vector may have little physical meaning. However, a qualitative comparison of the multichannel and single-record methods can be very useful, as each method can yield different insights upon visual inspection. In addition, the differences in splitting behavior between station TKA and station SGN suggest that a quantitative comparison of the single-measurement methods (SC1991 and
LMP1999) can increase confidence in individual splitting measurements for stations in regions that may overlie complex geological structures. Discrepancies between measured splitting parameters outside the $2 \sigma$ confidence intervals indicate that (1) complex anisotropy is likely, and that good coverage in incoming polarization angle is needed to fully characterize the anisotropy, and that (2) the individual records exhibiting discrepancies should not be included in the interpretation of the splitting parameters. This allows the analyst to discard questionable measurements and thus instills more confidence in the results.

In the case of the stations discussed in this article, the combination of measurement methods and good data coverage allow us to characterize the anisotropic structure beneath TKA and SGN with a high degree of confidence. Station TKA, which is located at the northernmost part of the Ryukyu arc (Figure 2), exhibits a splitting pattern that is consistent with a single anisotropic layer, and we find a fast direction that trends northeast-southwest. This direction, 
(b)
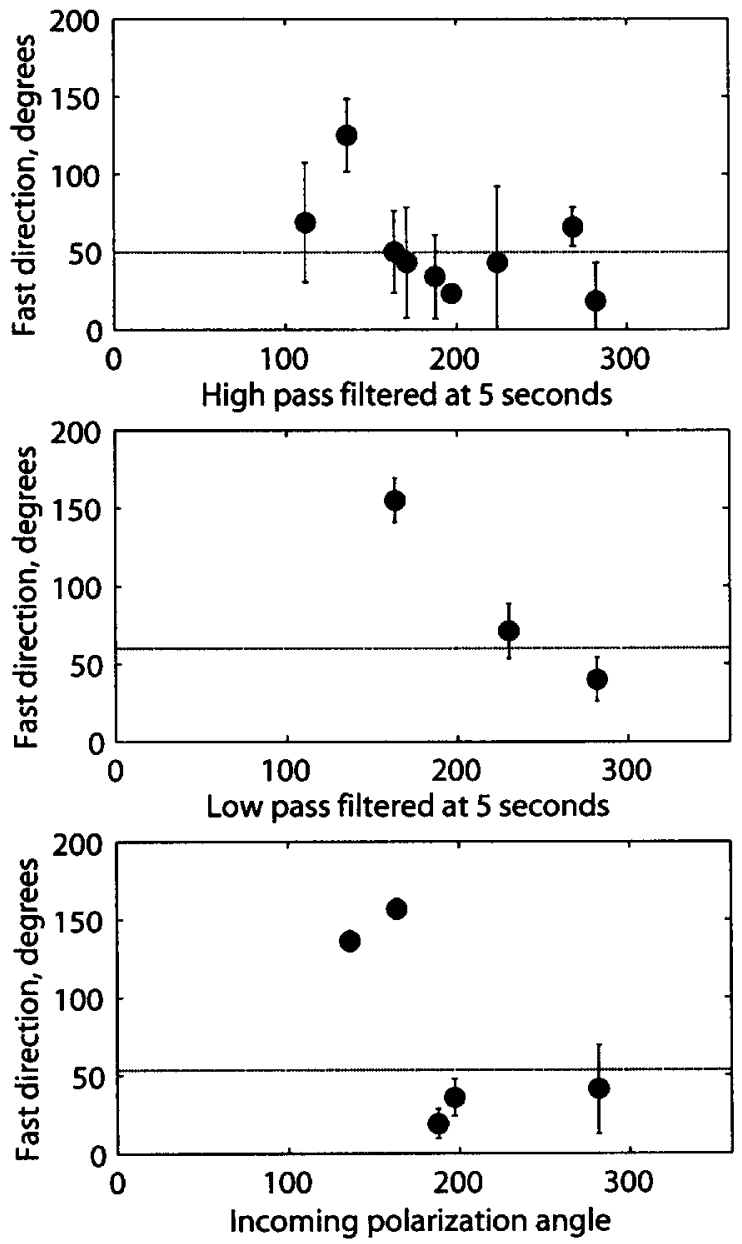

Filtered between 8 and 50 seconds
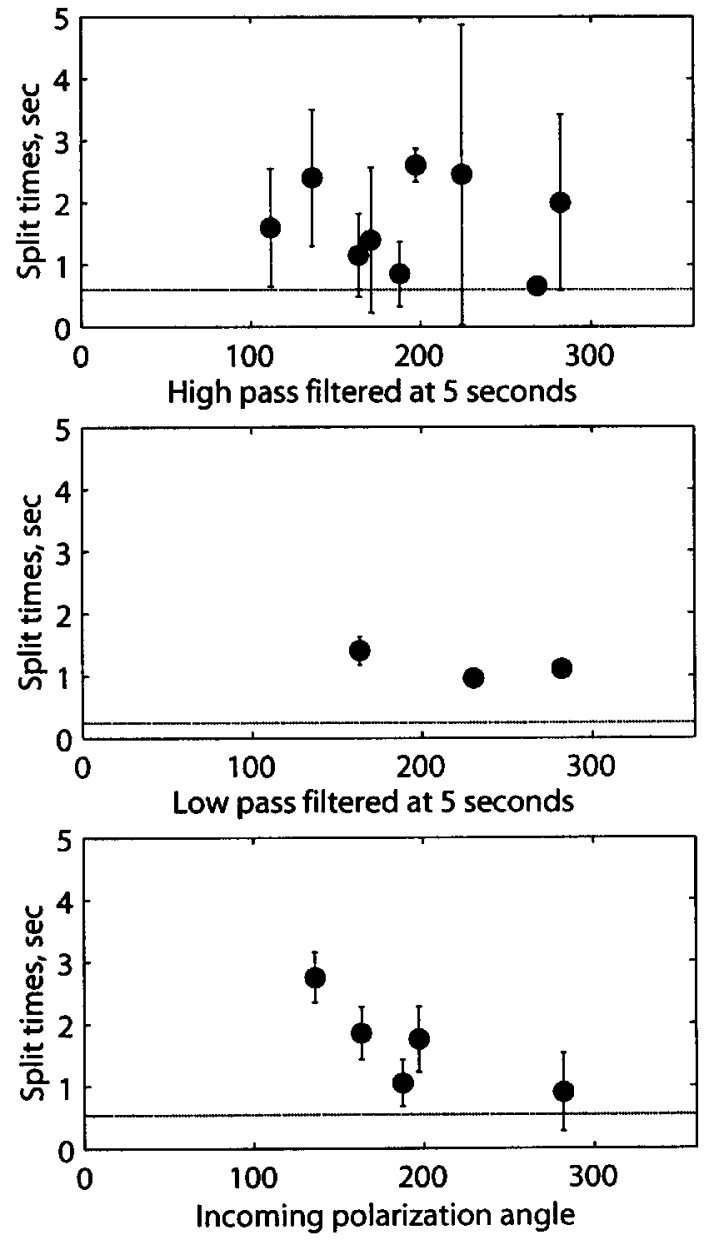

Figure 9. (continued) (b) Results of filter tests with the SC1991 method for each of the filtering schemes discussed above. The horizontal lines on each plot indicate the average splitting parameters obtained with the C2000 method for each frequency band.

(continued)

parallel to the strike of the Ryukyu trench and perpendicular to the direction of convergence at the Ryukyu arc, is consistent with the fast directions at other Ryukyu arc stations (Long and van der Hilst, 2005). Station SGN is located in a more tectonically complicated region and overlies a region of complicated slab morphology (see Long and van der Hilst, 2005, and references therein) The more complicated splitting pattern exhibited at station SGN, and the existence of discrepancies among the splitting measurement methods at this station, are consistent with a two-layer anisotropic model.

\section{Summary}

We have evaluated the performance of three different methods of estimating shear-wave splitting parameters from broadband recordings from the two considered Japanese stations. We generally find that the three methods agree well with each other, although the presence of complex aniso- tropy (inferred from backazimuthal variations in apparent splitting parameters) beneath the seismic station can introduce discrepancies among the methods. The Silver and Chan (1991) method seems particularly affected by the presence of complex anisotropy beneath the station. We find that the multichannel method of Chevrot (2000) is more robust when the data are subjected to different filters than the singlerecord methods of Silver and Chan (1991) and Levin et al. (1999). We conclude that a combination of multichannel and single-record methods can serve as a powerful tool for characterizing anisotropy beneath a seismic station, especially in the presence of anisotropic structure that is more complex than a simple single, horizontal anisotropic layer model. Specifically, a comparison among the three methods can help identify stations that overlie anisotropic structure that is more complex than a simple, horizontal anisotropic layer, and the analysis of discrepancies between single-record SC1991 and LMP1999 measurement methods can help identify individual measurements that are unreliable. 
(c)
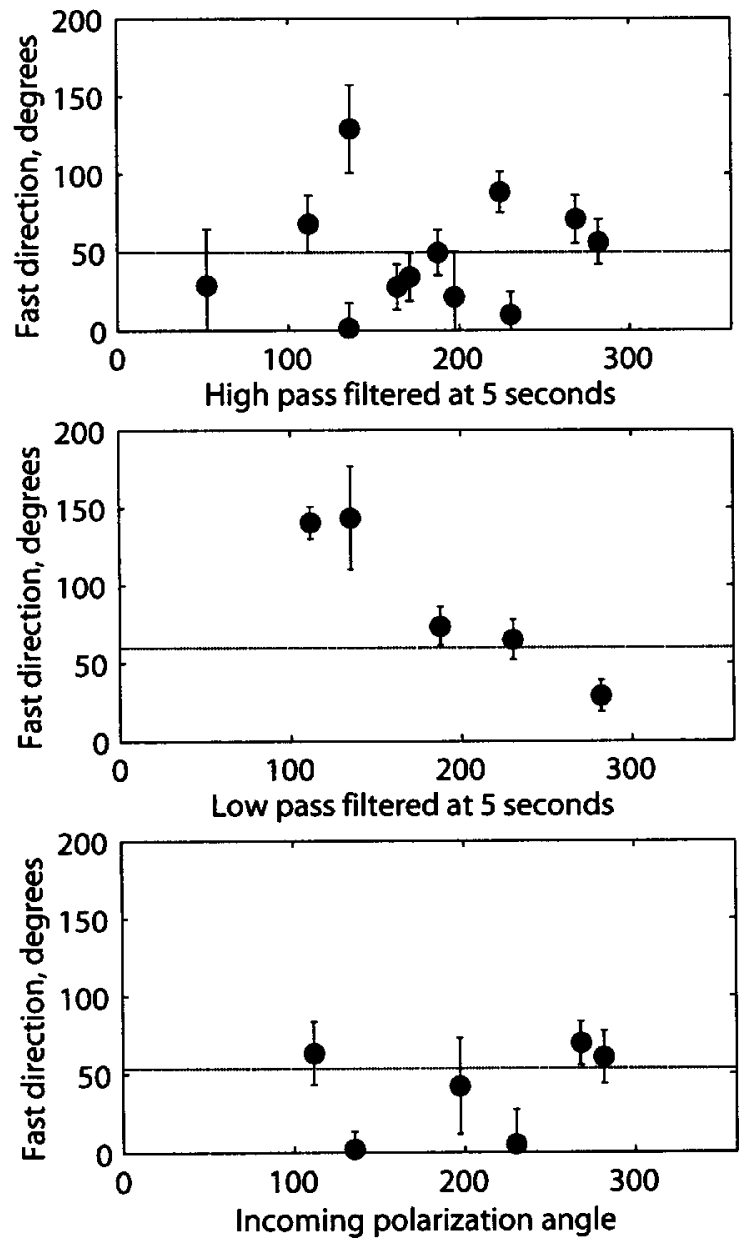

Filtered between 8 and 50 seconds
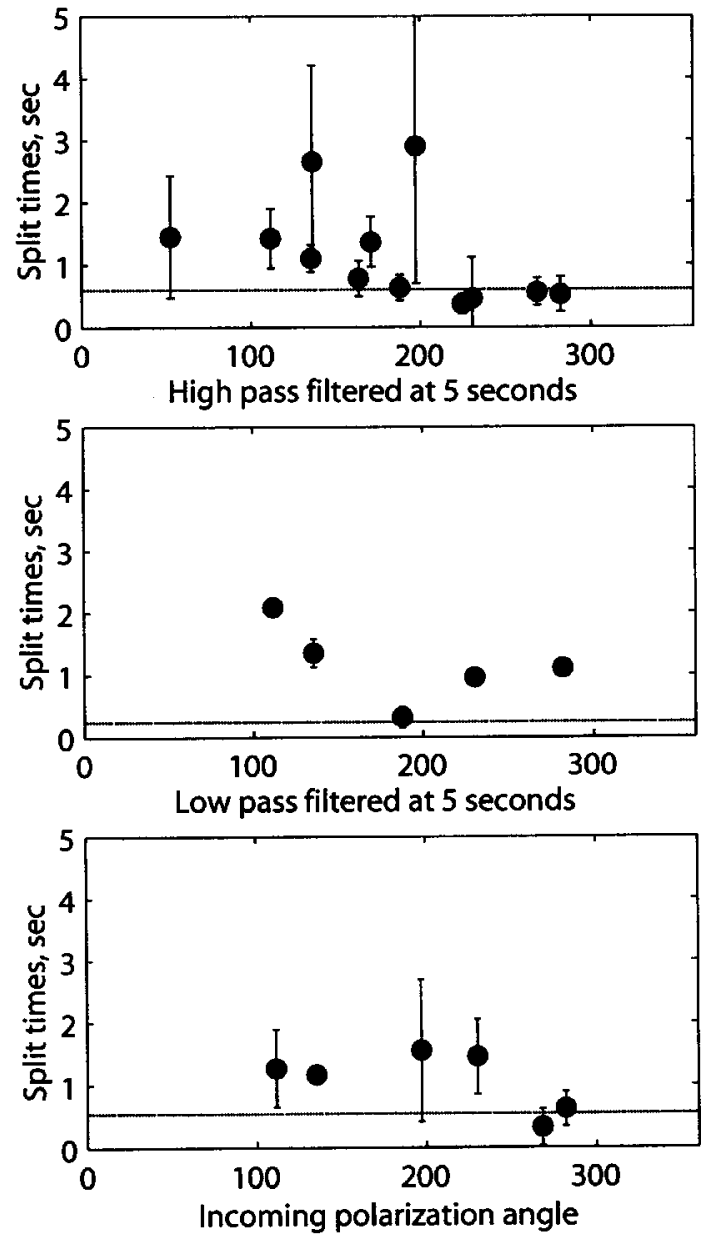

Figure 9. (continued) (c) Results of filter tests with the LMP1999 method for each of the filtering schemes discussed above. The horizontal lines on each plot indicate the average splitting parameters obtained with the C2000 method for each frequency band.

\section{Acknowledgments}

We thank Sebastien Chevrot for assistance with data processing and for providing his splitting codes, and Bill Menke for making his crosscorrelation code freely available. We thank the Japanese National Research Institute for Earth Science and Disaster Prevention for maintaining the $F$ net array and making the data freely available and easily accessible. This article was greatly improved by insightful comments from Matt Fouch and an anonymous reviewer. This research was supported by NSF grant EAR0337697 and by an NSF Graduate Research Fellowship awarded to M.D.L.

\section{References}

Ando, M., Y. Ishikawa, and F. Yamazaki (1983). Shear wave polarization anisotropy in the upper mantle beneath Honshu, Japan. J. Geophys. Res. 88, 5850-5864.

Babuška, V., and M. Cara (1991). Seismic Anisotropy in the Earth, Modern Approaches in Geophysics, vol. 10, Kluwer Academic Publishers, Dordrecht, The Netherlands.

Bowman, J. R., and M. Ando (1987). Shear-wave splitting in the uppermantle wedge above the Tonga subduction zone, Geophys. J. R. Astr. Soc. 88, 25-41.
Chevrot, S. (2000). Multichannel analysis of shear wave splitting, J. Geophys. Res. 105, 21,579-21,590.

Clitheroe, G., and R. van der Hilst (1998). Complex anisotropy in the Australian lithosphere from shear-wave splitting in broad-band SKS records, in Structure and Evolution of the Australian Continent, Geodyn. Ser., vol. 26, J. Braun, J. Dooley, B. Goleby, R. van der Hilst, and C. Klootwijk (Editors), AGU, Washington, D.C., 73-78.

Forsyth, D. W. (1975). The early structural evolution and anisotropy of the oceanic upper mantle, Geophys. J. R. Astr. Soc. 43, 103-162.

Fukao, Y. (1984). Evidence from core-reflected shear waves for anisotropy in the Earth's mantle, Nature 309, 695-698.

Keith, C. M., and S. Crampin (1977). Seismic body waves in anisotropic media: synthetic seismograms, Geophys. J. R. Astr. Soc. 94, 225-243.

Levin, V., D. Droznin, J. Park, and E. Gordeev (2004). Detailed mapping of seismic anisotropy with local shear waves in southeastern Kamchatka, Geophys. J. Int. 158, 1009-1023.

Levin, V., W. Menke, and J. Park (1999). Shear wave splitting in the Appalachians and the Urals: a case for multilayered anisotropy, J. Geophys. Res. 104, 17,975-17,993.

Long, M. D., and R. D. van der Hilst (2005). Upper mantle anisotropy and deformation beneath Japan from shear wave splitting, Phys. Earth Planet. Interiors, in press.

Matcham, I., M. K. Savage, and K. R. Gledhill (2000). Distribution of 
seismic anisotropy in the subduction zone beneath the Wellington region, New Zealand, Geophys. J. Int. 140, 1-10.

Menke, W., and V. Levin (2003). The cross-convolution method for interpreting SKS splitting observations, with application to one- and twolayer anisotropic earth models, Geophys. J. Int. 154, 379-392.

Özaleybey, S., and M. K. Savage (1994). Double-layer anisotropy resolved from $S$ phases, Geophys. J. Int. 117, 653-664.

Park, J., and V. Levin (2002). Seismic anisotropy: tracing plate dynamics in the mantle, Science 296, 485-489.

Restivo, A., and G. Helffrich (1999). Teleseismic shear wave splitting measurements in noisy environments, Geophys. J. Int. 137, 821-830.

Rumpker, G., and P. G. Silver (1998). Apparent shear-wave splitting parameters in the presence of vertically varying anisotropy, Geophys. $J$. Int. 135, 790-800.

Savage, M. K. (1999). Seismic anisotropy and mantle deformation: what have we learned from shear wave splitting? Rev. Geophys. 37, 65106.

Savage, M. K., and P. G. Silver (1993). Mantle deformation and tectonics: constraints from seismic anisotropy in the western United States, Phys. Earth Planet. Interiors 78, 207-227.

Schulte-Pelkum, V., and D. Blackman (2003). A synthesis of $P$ and $S$ anisotropy, Geophys. J. Int. 154, 166-178.

Silver, P. G. (1996). Seismic anisotropy beneath the continents: probing the depths of geology, Annu. Rev. Earth Planet. Sci. 24, 385-432.

Silver, P. G., and W. W. Chan (1988). Implications for continental structure and evolution from seismic anisotropy, Nature 335, 34-39.
Silver, P. G., and W. W. Chan (1991). Shear wave splitting and subcontinental mantle deformation, J. Geophys. Res. 96, 16,429-16,454.

Silver, P. G., and M. K. Savage (1994). The interpretation of shear-wave splitting parameters in the presence of two anisotropic layers, Geophys. J. Int. 119, 949-963.

Simons, F. J., R. D. van der Hilst, J.-P. Montagner, and A. Zielhuis (2002). Multimode Rayleigh wave inversion for heterogeneity and azimuthal anisotropy of the Australian upper mantle, Geophys. J. Int. 151, 738 754.

Vidale, J. E. (1986). Complex polarization analysis of particle motion, Bull. Seism. Soc. Am. 71, 1511-1530.

Vinnik, L. P., V. Farra, and B. Romanowicz (1989). Azimuthal anisotropy in the Earth from observations of SKS at GEOSCOPE and NARS broadband stations, Bull. Seism. Soc. Am. 79, 1542-1558.

Wolfe, C. J., and P. G. Silver (1998). Seismic anisotropy of oceanic upper mantle; shear wave splitting methodologies and observations, J. Geophys. Res. 103, 749-771.

Department of Earth, Atmospheric, and Planetary Sciences

Massachusetts Institute of Technology

77 Massachusetts Avenue

Cambridge, Massachusetts 02139

mlong@mit.edu

hilst@mit.edu

Manuscript received 8 June 2004. 